\title{
FIXED POINTS OF TOPOLOGICALLY STABLE FLOWS
}

BY

MIKE HURLEY

\begin{abstract}
This paper concerns certain necessary conditions for a flow to be topologically stable (in the sense of $\mathrm{P}$. Walters). In particular, it is shown that under fairly general conditions one can conclude that a topologically stable flow has a finite number of fixed points, and each of these is isolated in the chain recurrent set of the flow.
\end{abstract}

The purpose of this paper is to describe extensions of some of the results of [14], which dealt with topologically stable diffeomorphisms on a compact manifold $M$. (Here "topologically stable" is used in the sense of P. Walters [22].) The current paper is concerned with properties of topologically stable flows. The main result is

THEOREM. Suppose that $f$ is a topologically stable flow on a compact manifold and that $X$ is a chain component of $f$. Then any two hyperbolic closed orbits of $f$ that are contained in $X$ have the same index.

Throughout this paper we will say that an orbit of a flow is closed if it is either a fixed point of the flow or a (nontrivial) periodic orbit. The index of a hyperbolic closed orbit is the pair $(s, u)$, where $s$ is the dimension of the stable manifold of the orbit and $u$ is the dimension of the unstable manifold of the orbit.

The techniques of the proof of the theorem yield two corollaries.

COROLlARY A. If $f$ is a topologically stable flow on a compact manifold $M$, then any hyperbolic fixed point of $f$ is isolated in the chain recurrent set of $f$ if either of the following conditions is met:

(1) $f$ is Kupka-Smale, or

(2) the dimension of $M$ is 3 .

COROLlaRY B. None of the geometric models of the Lorenz attractor is topologically stable.

1. Background and definitions. Throughout this paper $M$ will denote a compact Riemannian manifold, and $X(M)$ will denote the set of $C^{1}$ flows on $M$, $X(M)=\{f: \mathbf{R} \times M \rightarrow M \mid f(0, x)=x, f(s+t, x)=f(s, f(t, x)), s, t \in \mathbf{R}, x \in M\}$. We give $X(M)$ the topology generated by the $C^{0}$ metric on generating vector fields

$$
d_{0}(f, g)=\sup \left\{\left\|f^{\prime}(0, x)-g^{\prime}(0, x)\right\| \mid x \in M\right\} .
$$

A flow $g$ is semiconjugate to a second flow $f$ if there is a continuous surjection $h: M \rightarrow M$ that takes oriented flow lines of $g$ to oriented flow lines of $f$. To be precise, this means that there is a continuous map $\tau: \mathbf{R} \times M \rightarrow M$ satisfying

$$
h(g(t, x))=f(\tau(t, x), x)
$$

Received by the editors December 19, 1983.

1980 Mathematics Subject Classification. Primary 58F10, 58F25, 58F13. 
for all $x$ and $t$, and such that for each $x$ in $M$, the map $\tau(-, x)$ is an orientation preserving homeomorphism from $\mathbf{R}$ to $\mathbf{R}$. In this situation we say that the pair $(h, \tau)$ is a semiconjugacy from $g$ to $f$; by abuse of notation we will sometimes omit explicit reference to the family of homeomorphisms $\tau$ and say that $h$ is the semiconjugacy from $g$ to $f$.

A $C^{1}$ flow $f$ is topologically stable if there is a neighborhood $U$ of $f$ in $X(M)$ (topologized as above) such that

(1) for each $g$ in $U$ there is a semiconjugacy $\left(h_{g}, \tau_{g}\right)$ from $g$ to $f$, and

(2) $\sup \left\{d\left(h_{g}(x), x\right) \mid x\right.$ in $\left.M\right\} \rightarrow 0$ as $d_{0}(g, f)=0$.

(Here $d(-,-)$ is a metric on $M$ induced by the Riemannian metric.) The only known examples of topologically stable flows are topologically equivalent to Axiom A flows $[15,16,19]$. (A topological equivalence is a semiconjugacy where the map $h: M \rightarrow M$ is a homeomorphism.) We do not repeat the definition of Axiom A for flows; this definition can be found in many places, for instance in [20] or in Chapter 7 of [4]. There have been several results indicating that any topologically stable flow must closely resemble one of these known examples $[\mathbf{7}, \mathbf{1 3}]$.

Central to much of the analysis of topologically stable flows is the notion of chain recurrence. If $C, \alpha$ are strictly positive constants, then a sequence $\left(t_{i}, x_{i}\right)$ in $\mathbf{R} \times M$ is called an $(\alpha, C)$-chain for $f$ if

(1) $t_{i} \geq C$ for each $i$, and

(2) $d\left(x_{i+1}, f\left(t_{i}, x_{i}\right)\right)<\alpha$ and each $i$.

A point $x$ in $M$ is in the chain recurrent set of $f$ if for any fixed $C>0$ and each $\alpha>0$ there is a finite sequence $\left(t_{i}, x_{i}\right), 0 \leq i \leq n(n>0)$ satisfying (1), (2), and

(3) $x_{0}=x_{n}=x$.

That is, $x$ is chain recurrent for $f$ if for some $C$ and each $\alpha>0$ there is an $(\alpha, C)$ chain that begins and ends at $x$. This definition is independent of which constant $C>0$ is chosen [12]. We denote the chain recurrent set of $f$ as $\operatorname{CR}(f)$. The chain recurrent set was introduced by Conley; for further details see [5].

There is a natural equivalence relation defined on $\operatorname{CR}(f): x \sim y$ if and only if for some $C>0$ and each $\alpha>0$ there exist two $(\alpha, C)$-chains, one beginning at $x$ and ending at $y$, and the other beginning at $y$ and ending at $x$. Equivalence classes under this equivalence relation are called chain components of $f$. It turns out for flows on compact manifolds that the chain components are exactly the connected components of $\mathrm{CR}(f)[6]$.

LEMMA 1. A subset $X$ of $M$ is a chain component of $f$ if and only if $X$ is a connected component of $\mathrm{CR}(f)$.

ProOF. (i) Claim: every chain component is connected. To see this let $X$ denote the chain component in question and suppose that $U$ and $V$ are open, disjoint, and cover $X$. For $x$ in $X$ define $U(x)=\{t \mid f(t, x) \in U\}$ and $V(x)=\{t \mid f(t, x) \in V\}$. Then $U(x)$ and $V(x)$ are open, disjoint, and cover $\mathbf{R}$, so for each $x$ at least one of them is empty. It follows that the sets $U \cap X$ and $V \cap X$ are $f$-invariant open and closed subsets of $X$. Since these sets are compact and disjoint, there is a positive constant $\delta$ such that no point of $U \cap X$ is within $\delta$ of any point of $V \cap X$. This fact, combined with the $f$-invariance of these two sets, shows that there cannot be a $(\delta, 1)$-chain $\left(t_{i}, x_{i}\right), 0 \leq i \leq n$, with the properties

(a) $x_{0} \in U \cap X$ and $x_{n} \in V \cap X$, 
(b) $x_{i} \in X$ for each $i$.

On the other hand, it can be shown that if two points lie in the same chain component of $f$, then for each $\delta>0$ there is a $(\delta, 1)$-chain contained in the given chain component that goes from $x$ to $y$. This last assertion can be proved by a simple extension of the arguments in [5, p. 38 and 19, p. 429]. This conclusion is incompatible with the nonexistence of a $(\delta, 1)$-chain satisfying (a) and (b) unless one of the two sets $U \cap X, V \cap X$ is empty. Hence $X$ is connected.

(ii) Claim: if $S$ is a connected component of $\mathrm{CR}(f)$, then $S$ is contained in a single chain component of $f$. To see this, let $\delta>0$ be given. $S$ is connected and compact, so we can find a finite sequence $\left(x_{i}\right), 0 \leq i \leq n$, such that

(c) $x_{0}=x_{n}$,

(d) $d\left(x_{i-1}, x_{i}\right)<\delta, 1 \leq i \leq n$,

(e) any point of $S$ is within $\delta$ of one of the $x_{i}$ 's.

By assumption each of the points $x_{i}$ is chain recurrent, so for each $i$ there is a finite $(\delta, 1)$-chain $C_{i}$ that begins and ends at $x_{i}$. If $C_{i}=\left\{\left(t_{i j}, x_{i j}\right) \mid 0 \leq j \leq N_{i}\right\}$, then define $C_{i}^{*}$ by $C_{i}^{*}=\left\{\left(t_{i j}, x_{i j}\right) \mid 0 \leq j<N_{i}\right\}$. The sequence $C$ defined by concatenating the $(\delta, 1)$-chains $C_{0}^{*}, C_{1}^{*}, \ldots, C_{n-1}^{*}, C_{n}$ is easily seen to be a $(2 \delta, 1)$ chain that begins and ends at $x_{0}$ and that contains all of the points $x_{i}$. Since the collection $\left\{x_{i}\right\}$ was chosen to be $\delta$-dense in $S$ it is not difficult to modify $C$ to show that for any given $x$ in $X$ there is a periodic $(3 \delta, 1)$-chain that begins and ends at $x_{0}$ and that contains $x$. Since $\delta$ was chosen arbitrarily, this establishes the claim.

Given a flow $f$ on $M$ and a point $x$ in $M$, define the stable set of $x, W^{\mathrm{s}}(x)$, and the unstable set of $x, W^{\mathrm{u}}(x)$, by

$$
\begin{aligned}
& W^{\mathrm{s}}(x)=\{y \text { in } M \mid d(f(t, x), f(t, y)) \rightarrow 0 \text { as } t \rightarrow \infty\}, \\
& W^{\mathrm{u}}(x)=\{y \text { in } M \mid d(f(t, x), f(t, y)) \rightarrow 0 \text { as } t \rightarrow-\infty\} .
\end{aligned}
$$

If $\alpha>0$, then one can also define the $\alpha$-local stable set of $x, W_{\alpha}^{\mathrm{s}}(x)$, and the $\alpha$-local unstable set of $x, W^{\mathrm{u}}(x)_{\alpha}$ by

$$
\begin{aligned}
& W_{\alpha}^{\mathrm{s}}(x)=\left\{y \text { in } W^{\mathrm{s}}(x) \mid d(f(t, x), f(t, y))<\alpha \text { for all } t \geq 0\right\} \\
& W_{\alpha}^{\mathrm{u}}(x)=\left\{y \text { in } W^{\mathrm{u}}(x) \mid d(f(t, x), f(t, y))<\alpha \text { for all } t \leq 0\right\} .
\end{aligned}
$$

If $\gamma$ is a closed orbit of $f$, then one can also define the stable and unstable sets of $\gamma$ as

$$
\begin{aligned}
& W^{\mathrm{s}}(\gamma)=\{y \text { in } M \mid d(f(t, y), \gamma) \rightarrow 0 \text { as } t \rightarrow \infty\} \\
& W^{\mathrm{u}}(\gamma)=\{y \text { in } M \mid d(f(t, y), \gamma) \rightarrow 0 \text { as } t \rightarrow-\infty\}
\end{aligned}
$$

Note that the stable (unstable) set of a periodic orbit contains the stable (unstable) set of each of the points on the orbit, but that it is not necessarily equal to the union of the stable (unstable) sets of the points on the orbit.

When we need to emphasize the dependence of any of these sets on the flow $f$, we will write them as $W^{\mathrm{s}}(x ; f)=W^{\mathrm{s}}(x)$, etc.

One other concept that we will be using is that of hyperbolicity of closed orbits. If $p$ is a fixed point of a flow $f$, then $p$ is said to be hyperbolic if the linear mapping $\partial f / \partial x(0, p): T M_{p} \rightarrow T M_{p}$ has no eigenvalue with real part 0 . In this case $W^{\mathrm{s}}(p)$ and $W^{\mathrm{u}}(p)$ are immersed submanifolds of $M$ whose dimensions sum to the dimension of $M[\mathbf{1 8}]$. There is a similar notion of hyperbolicity for periodic orbits (other than fixed points) of $f$. If $\gamma$ is a hyperbolic periodic orbit of $f$, 
then $W^{\sigma}(\gamma)=\left\{\bigcup W^{\sigma}(q) \mid q \in \gamma\right\}, \sigma=\mathrm{s}$ or $\mathrm{u}$, and $W^{\mathrm{s}}(\gamma), W^{\mathrm{u}}(\gamma)$ are immersed submanifolds whose dimensions sum to one plus the dimension of $M$ [18].

An important class of flows is the collection of Kupka-Smale flows. $f$ is KupkaSmale if two conditions are met:

(a) every closed orbit of $f$ is hyperbolic,

(b) any intersection of stable and unstable manifolds of closed orbits is transverse. The collection of Kupka-Smale flows is $C^{1}$ dense in the set of all $C^{1}$ flows on $M$ $[20,18]$.

2. Basic results. In this section we give a brief outline of the extension of certain results from $[\mathbf{1 4}]$ to the flow case. There are two basic facts underlying these results. The first of these is the $C^{0}$ density of the set of Smale flows $[\mathbf{1 7}$, 25]. (A flow $f$ is a Smale flow if it satisfies three conditions: (i) $\operatorname{CR}(f)$ has a hyperbolic structure; (ii) each chain component of $f$ is either one-dimensional or is a single point; and (iii) $f$ satisfies the strong transversality condition. See $[8]$ for more details.)

The second underlying fact is the fairly complete understanding of the dynamics of the restriction of a Smale flow to any of its chain components $[\mathbf{2}, \mathbf{3}]$. In particular, the following properties are known.

2.1 If $f$ is a Smale flow, then $\operatorname{CR}(f)$ is equal to the closure of the set of closed orbits of $f$. (Recall that a closed orbit is either a fixed point or a periodic orbit.) Moreover, all of the closed orbits of $f$ are hyperbolic.

2.2 If $f$ is a Smale flow, then for sufficiently small $\alpha>0$ there is a $\beta>0$ with the property that if $x, y$ are in $\operatorname{CR}(f)$ and $d(x, y)<\beta$, then there is a constant $\nu=\nu(x, y)$ with $|\nu| \leq \alpha$ and with $W_{\alpha}^{\mathrm{s}}(f(\nu, x)) \cap W_{\alpha}^{\mathrm{u}}(y)$ equal to exactly one point $z$ with $z$ in $\mathrm{CR}(f)$. $\beta$ can be chosen small enough to ensure that $x, y$ lie on a single chain component of $f$; in this case $z$ also lies on that chain component $[\mathbf{3}, \mathbf{4}, \mathbf{1}]$.

2.3 If $f$ is a Smale flow and $X$ is a chain component of $f$, then for any two points $x, y$ in $X$,

$$
\left(\bigcup\left\{W^{\mathrm{s}}(f(t, x)) \mid t \text { in } \mathbf{R}\right\}\right) \cap\left(\bigcup\left\{W^{\mathrm{u}}(f(t, y)) \mid t \text { in } \mathbf{R}\right\}\right)
$$

is dense in $X$ [1, Lemma 1.5 and Theorem 3.2].

(The chain components of a Smale flow are usually called basic sets.)

We will also use the following facts from $[\mathbf{1 3}]$.

2.4 If $f$ is topologically stable and $X$ is a chain component of $f$, then

(a) $X$ is the closure of the set of points lying on closed orbits in $X$.

(b) The restriction of $f$ to $X$ is topologically transitive; that is, there is a point $x$ in $X$ such that the forward and the backward $f$-orbits of $x$ are dense in $X$.

(c) There are only finitely many chain components of $f$.

3. Proof of the main results. In what follows we will be using the notions of the alpha-limit set and the omega-limit set of a point $x$ under a flow $f$. We remind the reader of the definition of these sets: $y$ is in the alpha-limit (omegalimit) set of $x$ if there is a sequence of real numbers $t_{n}$ with $t_{n} \rightarrow-\infty\left(t_{n} \rightarrow \infty\right)$ and $f\left(t_{n}, x\right) \rightarrow y$. Since $M$ is compact each of these sets is nonempty, closed, $f$-invariant, and chain recurrent $[\mathbf{1 1}]$. 
PROPOSITION 2. Suppose that $f$ is a topologically stable flow and that $X$ is a chain component of $f$. Let $g$ be a second flow on $M$ and $(h, \tau)$ a semiconjugacy from $g$ to $f$. Then

(a) $h(\mathrm{CR}(g))=\mathrm{CR}(f)$.

(b) $h^{-1}(X)$ contains every chain component of $g$ that it meets.

(c) $g$ has a chain component $Y$ with $h(Y)=X$.

PROOF. Let a constant $\varepsilon>0$ be given, and choose $\delta>0$ small enough that $d(x, y)<\delta$ implies that $d(h(x), h(y))<\varepsilon$. For each $x$ in $M$ the map $\tau(-, x)$ is an orientation preserving homeomorphism from $\mathbf{R}$ to $\mathbf{R}$, so there is a positive constant $C$ such that $\tau(C, x)>0$ for each $x$ in $M$; by compactness of $M$ and continuity of $\tau$ it follows that there is a constant $T$ satisfying $\tau(C, x) \geq T>0$ for all $x$ in $M$. Now it is easy to check that if $\left\{\left(x_{i}, t_{i}\right)\right\}$ is a $(\delta, C)$-chain for $g$, then $\left\{\left(h\left(x_{i}\right), \tau\left(t_{i}, x_{i}\right)\right)\right\}$ is an $(\varepsilon, T)$-chain for $f$ (recall that $h(g(t, x))=f(\tau(t, x), h(x)))$. Since $\varepsilon$ was arbitrary and $T$ is independent of $\varepsilon$, this shows that $h(\mathrm{CR}(g))$ is contained in $\mathrm{CR}(f)$; the fact that these two sets are equal will follow from (c). Assertion (b) is an immediate consequence of this inclusion and Lemma 1. The proof of $(c)$ is essentially the same as the proof of the analogous result in [14]. Use 2.4(b) to find a point $x$ in $X$ whose $f$-omega-limit set is all of $X$. Pick $y$ in $h^{-1}(x)$ and note that $h$ maps the $g$-omega-limit set of $y$ onto the $f$-omega-limit set of $x$. It is easy to see that the omega-limit set of any point is contained in a single chain component, so let $Y$ be the chain component of $g$ that contains the $g$-omega-limit set of $y$.

LEMMA 3. Suppose that $(h, \tau)$ is a semiconjugacy from $g$ to $f$ and that $\gamma$ is a closed orbit of $f$. If $p$ is in $h^{-1}(\gamma)$, then $h\left(W^{\sigma}(p ; g)\right) \subset W^{\sigma}(\gamma ; f)$ for $\sigma=\mathrm{s}$ or $\mathrm{u}$.

PROOF. If $y$ is in $W^{\mathrm{s}}(p ; g)$, then the distance between $g(t, y)$ and $g(t, p)$ goes to 0 as $t$ goes to infinity. As we have $h$ continuous, $h(g(t, x))=f(\tau(t, x), h(x))$, and $f(s, h(p)) \in \gamma$ for all $s$, we can conclude that

$$
d(f(\tau(t, y), h(y)), \gamma) \rightarrow 0 \quad \text { as } t \rightarrow \infty
$$

Since $\tau(-, y)$ is an orientation preserving homeomorphism of the real line, this establishes that $h(y)$ lies in $W^{\mathbf{s}}(\gamma ; f)$. The proof of the second case is essentially the same.

Proposition 4. Suppose $f$ is a topologically stable flow, $X$ is one of its chain components, and that $\gamma_{1}, \gamma_{2}$ are closed orbits of $f$ that lie in $X$. Then $W^{\mathrm{s}}\left(\gamma_{1} ; f\right) \cap$ $W^{\mathrm{u}}\left(\gamma_{2} ; f\right) \cap X$ is dense in $X$.

Proof. Begin by choosing a Smale flow $g$ that is semiconjugate to $f$. This is possible because of the $C^{0}$-density of the collection of Smale flows. Let $(h, \tau)$ denote the semiconjugacy from $g$ to $f$. By Proposition 2 there is a chain component (basic set) of $g, Y$, with $h(Y)=X$, so we can choose points $y_{j}$ in $Y \cap h^{-1}\left(\gamma_{j}\right)$ for $j=1$ and 2. If $Y$ is a single point, then so is $X$ and there is nothing to prove. Since $g$ is a Smale flow, the only other possibility is that $Y$ is a one-dimensional hyperbolic set for $g$. In this case it is known that the intersection of the stable set of any orbit in $Y$ with the unstable set of any other orbit in $Y$ is contained in $Y$ and dense in $Y$ (see 2.3). In particular, if we let $O\left(y_{j}\right)$ denote the $g$-orbit of $y_{j}$ for $j=1,2$, then the set $D=W^{\mathrm{s}}\left(O\left(y_{1}\right)\right) \cap W^{\mathrm{u}}\left(O\left(y_{2}\right)\right) \cap Y$ is dense in $Y$. Since $h: Y \rightarrow X$ is 
a continuous surjection, $h(D)$ is dense in $X$. Finally, Lemma 3 shows that $h(D)$ is contained in the subset of $X$ that the current proposition asserts is dense in $X$.

Recall that if $\gamma$ is a hyperbolic closed orbit of a flow $g$ on $M$, then $W^{\mathrm{s}}(\gamma)$ and $W^{\mathrm{u}}(\gamma)$ are immersed submanifolds of $M$. In this situation the index of $\gamma$ is defined to be the pair of integers $(S, U)$ where $S=\operatorname{dim}\left(W^{\mathrm{s}}(\gamma)\right)$ and $U=\operatorname{dim}\left(W^{\mathrm{u}}(\gamma)\right)$. If $\gamma$ is a hyperbolic fixed point $p$, then $S+U=\operatorname{dim}(M)$; if $\gamma$ is a nontrivial hyperbolic periodic orbit, then $S+U=\operatorname{dim}(M)+1[\mathbf{1 8}]$.

Proposition 5. Suppose that $f$ is topologically stable, $X$ is one of the chain components of $f$, and that $\gamma_{1}, \gamma_{2}$ are hyperbolic closed orbits of $f$ contained in $X$. Then $\gamma_{1}$ and $\gamma_{2}$ have the same index.

ProOF. We assume that $\gamma_{1} \neq \gamma_{2}$. By the Hartman-Grobman theorem and the local stability of hyperbolic orbits $[\mathbf{1 8}, \mathbf{2 0}]$, there are disjoint neighborhoods $U_{j}$ of $\gamma_{j}$ in $M, j=1,2$, and a $C^{1}$ neighborhood $G$ of $f$ in $X(M)$ satisfying

(1) For $j=1$ and $j=2$, if $g$ is in $G$ then $g$ has a hyperbolic closed orbit $\lambda_{j}(g)$ contained in $U_{j}$ and with the index of $\lambda_{j}(g)$ equal to the index of $\gamma_{j}$.

(2) $\lambda_{j}(g)$ is the maximal $g$-invariant set in $U_{j}, \lambda_{j}(g)=\left\{x \mid g(t, x) \in U_{j}\right.$ for all $t$ in $\mathbf{R}$ \}. (Note that this forces $\lambda_{j}(f)=\gamma_{j}$.)

(3) Each $g$ in $G$ is $C^{0}$ close enough to $f$ that there is a semiconjugacy $h$ from $g$ to $f$ with $h$ close enough to the identity that $h^{-1}\left(\gamma_{j}\right) \subset U_{j}, j=1,2$.

$\gamma_{j}$ is closed and $f$-invariant, so $h^{-1}\left(\gamma_{j}\right)$ is closed and $g$-invariant; hence $(2)$ and (3) combine to show that $h^{-1}\left(\gamma_{1}\right)=\lambda_{j}(g)$ for each $g$ in $G, j=1,2$.

Since the set of Kupka-Smale flows is dense in the $C^{1}$ topology [18], we can find a Kupka-Smale flow $g$ in $G$. We use this particular choice of $g$ in the remainder of the proof. Let $\lambda_{j}=\lambda_{j}(g), j=1,2$. We claim that $W^{\mathrm{s}}\left(\lambda_{1}\right) \cap W^{\mathrm{u}}\left(\lambda_{2}\right)$ and $W^{\mathrm{u}}\left(\lambda_{1}\right) \cap W^{\mathrm{s}}\left(\lambda_{2}\right)$ are both nonvoid. To see this, use Proposition 4 to choose a point $x$ in $W^{\mathrm{s}}\left(\gamma_{1}\right) \cap W^{\mathrm{u}}\left(\gamma_{2}\right)$ and let $y$ be a point in $h^{-1}(x)$. As in the proof of Proposition 2, the semiconjugacy $h$ takes the $g$-omega-limit set of $y$ into the $f$ omega-limit set of $x$, which by choice of $x$ is equal to $\gamma_{1}$. Thus the $g$-omega-limit set of $y$ lies in $h^{-1}\left(\gamma_{1}\right)=\lambda_{1}$, and so $y$ is contained in $W^{\mathrm{s}}\left(\lambda_{1} ; g\right)$. A similar argument involving the $g$-alpha-limit set of $y$ shows that $y$ is also contained in $W^{\mathrm{u}}\left(\lambda_{2} ; g\right)$, so that $W^{\mathrm{s}}\left(\lambda_{1} ; g\right) \cap W^{\mathrm{u}}\left(\lambda_{2} ; g\right) \neq \varnothing$, as claimed. Reversing the roles of $\lambda_{1}$ and $\lambda_{2}$ completes the proof of the claim.

Now a simple dimension count suffices to finish the proof of the proposition. Since $g$ is Kupka-Smale the intersection of the stable manifold of any closed orbit of $g$ with the unstable manifold of any closed orbit of $g$ is necessarily transverse, and this intersection is either empty or is a submanifold of dimension at least one (this uses the assumption on $f$ that $\gamma_{1}$ and $\gamma_{2}$ are distinct orbits, so that $\lambda_{1}$ and $\lambda_{2}$ are also distinct orbits). The consequence of this fact and the claim is the following pair of inequalities. In what follows $\left(S_{j}, U_{j}\right)$ represents the index of $\lambda_{j}$ with respect to $g$, and $m=\operatorname{dim}(M)$.

(4) $U_{1}+S_{2} \geq m+1$,

(5) $U_{2}+S_{1} \geq m+1$.

In addition, general facts about indices of hyperbolic closed orbits give a second pair of inequalities

(6) $U_{1}+S_{1} \leq m+1$,

(7) $U_{2}+S_{2} \leq m+1$. 
Subtracting (6) from (4) shows that $S_{2} \geq S_{1}$; by the symmetry in the argument, we conclude that $S_{2}=S_{1}$. Similarly, a comparison of (6) and (5) will yield $U_{2}=U_{1}$. Combining these equalities with (1) gives the conclusion of the proposition.

COROLLARY 6. Using the notation of the proposition, if $X$ contains a hyperbolic fixed point, then $X$ contains no other hyperbolic closed orbit.

Proof. A comparison of the sum of (4) and (5) with the sum of (6) and (7) shows that each of (4)-(7) must in fact be an equality. If $\gamma_{1}$ were a fixed point and $\left(S_{1}, U_{1}\right)$ was its index, then $S_{1}+U_{1}=m$, not $m+1$. This contradicts the fact that (6) is an equality, so the assumption $\gamma_{1} \neq \gamma_{2}$ used in deriving (4) must be false.

COROLlARY 7. If a Kupka-Smale flow is topologically stable, then each of its fixed points is a chain component (that is, each of its fixed points is isolated from the rest of the chain recurrent set).

PROOF. If $f$ is topologically stable, then the closed orbits of $f$ are dense in $\mathrm{CR}(f)$, and if $f$ is Kupka-Smale then each of these closed orbits is hyperbolic, so the result follows directly from the previous corollary.

If the dimension of $M$ is 3 one can obtain the result of this last corollary without assuming that the topogically stable flow $f$ is Kupka-Smale. This is because of the fact that if $\operatorname{dim}(M)$ is 3 , then any hyperbolic fixed point is either an attractor (sink), a repeller (source), or else either its stable or its unstable manifold has dimension 1.

PROPOSITION 8. Suppose $f$ is topologically stable and that $X$ is a chain component of $f$ that contains a hyperbolic fixed point of $f$. If either the stable manifold or the unstable manifold of this fixed point is one-dimensional, then $X$ consists of that fixed point.

ProOF. Suppose for definiteness that $p$ is the fixed point, that $\operatorname{dim}\left(W^{\mathrm{u}}(p)\right)=1$, and that $\gamma$ is a closed orbit in $X$ other than $p$. The dimensionality assumption implies that $W^{\mathrm{u}}(p)$ consists of $p$ plus two other $f$-orbits. By Proposition 4 and the fact that any $f$-orbit is either disjoint from $W^{\mathbf{s}}(\gamma ; f)$ or else is contained in it we see that $W^{\mathrm{s}}(\gamma ; f)$ must contain at least one of the two orbits in $W^{\mathrm{u}}(p ; f)$ other than $p$. Combining this with the fact that the stable sets of distinct periodic orbits are disjoint shows that $X$ can contain at most two periodic orbits besides $p$. Since, as noted in 2.4, the closed orbits of $f$ in $X$ are dense in $X$ this shows that $X$ must consist of a finite number of closed orbits. But now the definition of a chain component implies that $X$ consists of a single closed orbit.

COROLlaRY 9. None of the geometric models of the Lorenz attractor is topologically stable.

ProOF. These models have been described in several places, notably $[\mathbf{9}, \mathbf{1 0}$, 23, 24, 21]. We will follow the description from [9]. The set of models consists of an open set $U_{0}$ in the set of $C^{1}$ vector fields on $S^{3}$ (open with respect to the $C^{1}$ topology on vector fields). Let $U$ denote the set of flows on $S^{3}$ that are generated by the vector fields in $U_{0}$. This set of flows has the property that there is an open subset $N$ of $S^{3}$ that is forward invariant under the flow of each $f$ in $U$ in the strong sense that $f(1, \operatorname{clos}(N)) \subset N$. Consequently $\bigcap_{t>0} f(t, N)$ is an attracting set $A_{f}$ of $f$. Additionally, for each $f$ in $U, A_{f}$ contains exactly one fixed point $p_{f}$ and 
this fixed point is a hyperbolic saddle. It is shown in the proofs of Theorems 12.1 and 12.2 of [9] that there is a dense subset $V$ of $U$ such that for each $g$ in $V A_{g}$ is connected and contained in $\operatorname{CR}(f)$. Applying Lemma 1 we see that $A_{g}$ is a chain component of $g$, so that each $g$ in $V$ has exactly one chain component contained in $N$. Because $f(1, \operatorname{clos}(N)) \subset N$ for each $f$ in $U$, it follows that any chain component of $f$ that meets $\operatorname{clos}(N)$ must be contained in $N$. Using this fact, the density of $V$ in $U$ and Proposition 2(c) one sees that if some $f$ in $U$ is topologically stable, then $f$ has a unique chain component in $N$. Denote this chain component by $X$, and note that $p_{f} \in X$. We claim that the fact that the topologically stable flow $f$ has only one chain component forces two incompatible conclusions; first, that $X=\left\{p_{f}\right\}$, and second, that $X=A_{f}$. (These are incompatible because $A_{f}$ is an attractor and $p_{f}$ is a saddle point.) The conclusion that $X=\left\{p_{f}\right\}$ is an immediate consequence of Proposition 8.

To see that $X=A_{f}$, note that $A_{f}$ is closed and $f$-invariant, so, if $x \in A_{f}$, then both its alpha-limit set and its omega-limit set are in $A_{f}$ and are chain recurrent. Since $X$ is the only chain component of $f$ in $A_{f}$, this forces both of these limit sets to be contained in $X$. It is easy to see that this forces $X$ to contain $x$, so that $A_{f} \subset X$. The reverse inclusion follows from the fact that $A_{f}$ is the maximal $f$-invariant set in $N$.

REMARK. In [9] J. Guckenheimer shows that the geometric models of the Lorenz attractor are not "topologically $\Omega$-stable", by which he means something very much different from the term "topologically stable" as it has been used in this paper. It is an unfortunate fact of life that "topological stability" is used in several quite distinct ways in the study of dynamical systems.

\section{REFERENCES}

1. R. Bowen, Periodic orbits for hyperbolic flows, Amer. J. Math. 94 (1972), 1-30.

2. __ One dimensional hyperbolic sets for flows, J. Differential Equations 12 (1972), 173179.

3. ___ Symbolic dynamics for hyperbolic flows, Amer. J. Math. 95 (1973), 429-460.

4. __ On Axiom A diffeomorphisms, C.B.M.S. Regional Conf. Ser. Math. no. 35, Amer. Math. Soc., Providence, R.I., 1978.

5. C. Conley, Isolated invariant sets and the Morse index, C.B.M.S. Regional Conf. Ser. Math., no. 38, Amer. Math. Soc., Providence, R.I., 1978.

6. _ The gradient structure of a flow, I.B.M. Res. RC 3932. Yorktown Heights, N.Y., 1972.

7. P. Fleming and M. Hurley, A converse topological stability theorem for flows on surfaces, J. Differential Equations 53 (1984), 172-191.

8. J. Franks, Homology and dynamical systems, C.B.M.S. Regional Conf. Ser. Math., no. 49, Amer. Math. Soc., Providence, R.I., 1982.

9. J. Guckenheimer, A strange, strange attractor, pp. 368-381 in J. Marsden, M. McCracken and G. Oster, The Hopf Bifurcation and its Applications, Springer-Verlag, New York, 1976.

10. J. Guckenheimer and R. F. Williams, Structural stability of Lorenz attractors, Publ. Inst. Hautes Ètudes Sci. 50 (1979), 59-72.

11. J. Hale, Ordinary differential equations, Wiley, New York, 1969.

12. M. Hurley, Attractors: persistence, and density of their basins, Trans. Amer. Math. Soc. 269 (1982), 247-271.

13. __ Consequences of topological stability, J. Differential Equations 54 (1984), 60-72.

14. __ Combined structural and topological stability are equivalent to Axiom A and the strong transversality condition, Ergodic Theory Dynamical Systems 4 (1984), 81-88. 
15. K. Kato and A. Morimoto, Topological stability of Anosov flows and their centralizers, Topology 12 (1973), 255-273.

16. __ Topological $\Omega$-stability of Axiom A flows with no $\Omega$-explosions, J. Differential Equations 34 (1979), 464-481.

17. M. M. C. de Oliveira, $C^{0}$-density of structurally stable vector fields, Bull. Amer. Math. Soc. 82 (1976), 786.

18. J. Palis and W. de Melo, Geometric theory of dynamical systems, Springer-Verlag, New York, 1982.

19. C. Robinson, Stability theorems and hyperbolicity in dynamical systems, Rocky Mountain J. Math. 7 (1977), 425-437.

20. S. Smale, Differentiable dynamical systems, Bull. Amer. Math. Soc. 73 (1967), 747-817.

21. C. Sparrow, The Lorenz equations: Bifurcation, chaos, and strange attractors, SpringerVerlag, New York, 1982.

22. P. Walters, Anosov diffeomorphisms are topologically stable, Topology 9 (1970), 71-78.

23. R. F. Williams, The structure of Lorenz attractors, Turbulence Seminar, Lecture Notes in Math., vol 615, Springer-Verlag, New York, 1977, pp. 94-112.

24. __ The structure of Lorenz attractors, Publ. Inst. Hautes Ètudes Sci. 50 (1979), 73-100.

25. E. C. Zeeman, Morse inequalities for diffeomorphisms with shoes and flows with solenoids, Dynamical Systems-Warwick 1974, Lecture Notes in Math., vol. 468, Springer-Verlag, New York, 1975, pp. 44-47.

Department of Mathematics and Statistics, Case Western Reserve UniverSity, CleVEland, OHIO 44106 